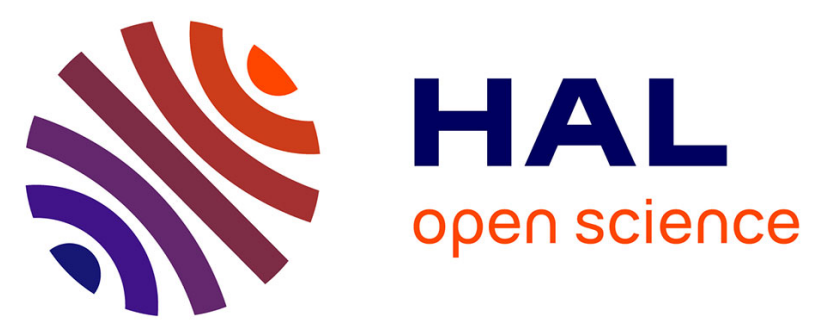

\title{
Structure of the monoclinic Al13Fe4(010) complex metallic alloy surface determined by low-energy electron diffraction
}

\author{
A. Matilainen, K. Pussi, R. D. Diehl, M. Hahne, P. Gille, É. Gaudry, L.N. \\ Serkovic Loli, G. Mcguirk, M.-C. De Weerd, V. Fournée, et al.
}

\section{To cite this version:}

A. Matilainen, K. Pussi, R. D. Diehl, M. Hahne, P. Gille, et al.. Structure of the monoclinic Al13Fe4(010) complex metallic alloy surface determined by low-energy electron diffraction. Physical Review B: Condensed Matter and Materials Physics (1998-2015), 2015, 10.1103/PhysRevB.92.014109 . hal-01218348

\section{HAL Id: hal-01218348 \\ https://inria.hal.science/hal-01218348}

Submitted on 21 Oct 2015

HAL is a multi-disciplinary open access archive for the deposit and dissemination of scientific research documents, whether they are published or not. The documents may come from teaching and research institutions in France or abroad, or from public or private research centers.
L'archive ouverte pluridisciplinaire HAL, est destinée au dépôt et à la diffusion de documents scientifiques de niveau recherche, publiés ou non, émanant des établissements d'enseignement et de recherche français ou étrangers, des laboratoires publics ou privés. 


\title{
Structure of the monoclinic $\mathrm{Al}_{13} \mathrm{Fe}_{4}(010)$ complex metallic alloy surface determined by low-energy electron diffraction
}

\author{
A. Matilainen, ${ }^{1}$ K. Pussi, ${ }^{1}$ R. D. Diehl,${ }^{2}$ M. Hahne, ${ }^{3}$ P. Gille,,${ }^{3}$ É. Gaudry, ${ }^{4}$ L. N. Serkovic Loli, ${ }^{4}$ G. M. McGuirk, ${ }^{4}$ \\ M.-C. de Weerd, ${ }^{4}$ V. Fournée, ${ }^{4}$ and J. Ledieu ${ }^{4}$ \\ ${ }^{1}$ Department of Mathematics and Physics, Lappeenranta University of Technology, P.O. Box 20 FIN-53851 Lappeenranta, Finland \\ ${ }^{2}$ Department of Physics, Penn State University, University Park, Pennsylvania 16802, USA \\ ${ }^{3}$ Department of Earth and Environmental Sciences, Crystallography Section, Ludwig-Maximilians-Universität München, Theresienstrasse 41, \\ D-80333 München, Germany \\ ${ }^{4}$ Institut Jean Lamour (UMR7198 CNRS, Université de Lorraine), Parc de Saurupt, 54011 Nancy Cedex, France
}

(Received 7 November 2014; revised manuscript received 29 April 2015; published 13 July 2015)

\begin{abstract}
Complex metallic alloys having isolated transition-metal elements in the surface layer have been reported to work well as selective hydrogenation catalysts. We report an experimental determination of the surface structure of one such compound $\mathrm{Al}_{13} \mathrm{Fe}_{4}(010)$. The structure was determined using low-energy electron diffraction. The best-fit structure terminates in a layer similar to the puckered bulk layer but lacking some of the $\mathrm{Al}$ and $\mathrm{Fe}$ atoms. Protruding Fe atoms are located in the middle of adjacent pentagonal $\mathrm{Al}$ formations, connected to each other by Al "glue" atoms. The top interlayer spacing is compressed relative to the bulk with oscillating relaxations observed for subsequent layers at the surface.
\end{abstract}

DOI: 10.1103/PhysRevB.92.014109

PACS number(s): 61.05.jh, 68.35.bd, 68.47.De

\section{INTRODUCTION}

Complex metallic alloys (CMAs) have long-range periodicity but often consist of the same local arrangements as the corresponding quasicrystalline phases. These so-called quasicrystal approximants are helpful in analyzing the structures of quasicrystals because they allow the use of traditional structure determination methods and can provide a starting model for the quasicrystal structure determination [1-3]. Some CMAs have also been found to offer a low-cost alternative for the design of selective and stable catalysts in heterogeneous catalysis [4]. The idea behind this site isolation concept is that small well-separated sites that contain an active transition-metal (TM) element reduce the number of possible reaction products by limiting the number of possible adsorption geometries. Recently, the $\mathrm{Al}_{13} \mathrm{Fe}_{4} \mathrm{CMA}$ has been identified as a promising complex intermetallic compound for use as an active and selective semihydrogenation catalyst [5]. In this paper, we present a structural investigation of the surface of this compound by quantitative low-energy electron diffraction under ultra-high-vacuum conditions.

Monoclinic $\mathrm{Al}_{13} \mathrm{Fe}_{4}$, which belongs to the space group $\mathrm{C} 2 / \mathrm{m}(\mathrm{mC} 102)$, is a four-layer approximant to the decagonal quasicrystal surface. Its structure is usually given as a stacking of pseudotenfold $(p-10 f)$ flat $(F)$ and puckered $(P)$ layers in a $F_{1} P_{1} F_{2} P_{2}$ sequence in the [010] direction. The puckered layers are mirrored against the $F_{2}$ layer. The flat layers are composed of $17 \mathrm{Al}$ atoms and $8 \mathrm{Fe}$ atoms, and the puckered layers are composed of $22 \mathrm{Al}$ and $4 \mathrm{Fe}$ atoms, so in total the unit cell contains 102 atoms. The lattice parameters are $a=15.492 \AA, b=8.078 \AA, c=12.471 \AA$, and $\beta=107.69^{\circ}$. The $F_{2}$ layer is the $F_{1}$ layer laterally shifted by $a / 2$, and the same is true for $P_{1}$ and $P_{2}$. Separation between the flat and the puckered layers is approximately $2 \AA$. The bulk unit cell is shown in Fig. 1.

The flat layer can be described as a tiling of rhombi and two different kinds of pentagons, whereas the puckered layer consists of pentagons and connecting "glue" atoms. These Al pentagons form bipentagonal motifs, related by the vertical position-above or below the mean plane position —of the Fe atoms that reside in the middle. This is illustrated in greater detail in Fig. 2. This interpretation conforms to the pentagonal bipyramid cluster model suggested by Henley [6] for the bulk structure. A cage compound interpretation has also been suggested based on quantum-mechanical calculations [7].

\section{STUCTURAL MODELS}

Based on the experimental results, the surface termination should consist of either the $F$ or the $P$ layer. A combination of both layers on the surface is ruled out by the scanning tunneling microscopy (STM) measurements performed by Ledieu et al.; the height of the step between the two consecutive terraces was found to be equal to half the lattice parameter $(b / 2)$ along the [010] direction [8]. For the structural determination, the flat and puckered bulk terminations were considered as trial structures, and incomplete variations of them were tested. In total, ten different trial structures were considered. Some of the incomplete variations are depicted in Fig. 3.

Based on the STM measurements, the most likely structural candidates were ones with protruding $\mathrm{Fe}$ atoms surrounded by $\mathrm{Al}$ pentagons on the top layer, but other structures were included in the analysis for validation purposes. Terminations with these $\mathrm{Al}$ bipentagonal motifs and protruding Fe atoms were the complete puckered layer, the puckered top layer [Fig. 3(a)], and the incomplete $A$ puckered layer [Fig. 3(d)].

\section{LEED EXPERIMENT, CALCULATIONS, AND RESULTS}

\section{A. Experiment}

The $\mathrm{Al}_{13} \mathrm{Fe}_{4}$ sample was grown from an Al-rich solution using the Czochralski method [9]. The crystal was oriented using backreflection Laue x-ray diffraction and was cut perpendicular to the [010] direction. Once mechanically polished, the sample was prepared under ultra-high-vacuum 


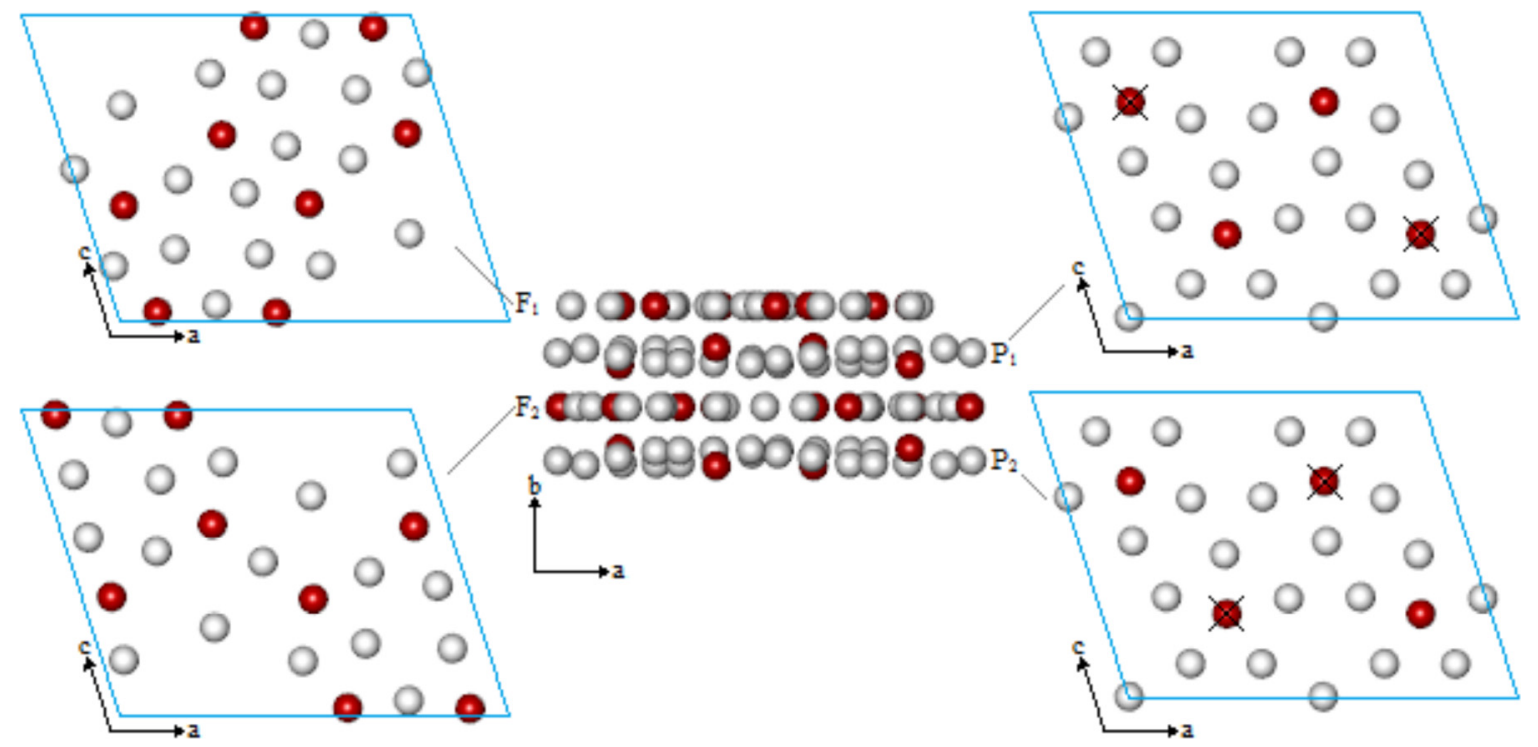

FIG. 1. (Color online) Side view of the bulk unit cell and top views of the individual layers. In the puckered layers, the Fe (red/dark) atoms that are below the mean plane position are indicated by crosses. The parallelograms denote the unit cell.

conditions (base pressure $5 \times 10^{-11}$ mbar). The $\mathrm{Al}_{13} \mathrm{Fe}_{4}(010)$ surface was prepared by $\mathrm{Ar}^{+}$ion sputtering at $1.5 \mathrm{keV}$ for $20 \mathrm{~min}$, after which it was annealed at $973 \mathrm{~K}$ for $90 \mathrm{~min}$. The temperature was measured using an infrared optical pyrometer with the emissivity set to 0.35 . This preparation led to a clean and flat surface as measured by x-ray photoelectron spectroscopy and scanning tunneling microscopy (see Ledieu et al. [8] for more preparation details). The low-energy electron diffraction (LEED) measurements were performed at $300 \mathrm{~K}$ using a three-grid retractable Omicron LEED apparatus equipped with an Ir/Th filament. The LEED images were recorded from 20 to $220 \mathrm{eV}$ with a $2-\mathrm{eV}$ step. Each image corresponds to an average of 60 frames. The intensities for each beam were extracted using the EASYLEED program $[10,11]$. The experimental data are provided in the Supplemental Material [12].

Figure 4 shows a LEED pattern from the surface at $300 \mathrm{~K}$. It is evident that there are two different reciprocal lattices present in this pattern; their lattice directions are denoted by red and blue vectors. The presence of two lattices indicates that there are crystalline twins present in the crystal — such planar defects are common in $\mathrm{Al}_{13} \mathrm{TM}_{4}$ structures [13]. The reciprocal lattice unit cell for each twin is shown by a parallelogram. It can be observed that the LEED spots corresponding to the blue reciprocal lattice are considerably brighter than those from the red lattice, indicating that there is significantly more of the blue lattice present on the region of the surface sampled by the LEED beam $(\sim 0.25$-mm diameter).

A total of 43 beams ranging in energy from 22 to $220 \mathrm{eV}$ and recorded at a sample temperature of $300 \mathrm{~K}$ were used for the experimental dataset for the two twins; 12 from one twin (blue), 7 from the other twin (red), and 24 with contributions from both twins (purple). For simplicity, we refer to the datasets and the twins by these color designations. Their energy ranges were $920 \mathrm{eV}$ (blue), $620 \mathrm{eV}$ (red), and $1898 \mathrm{eV}$ (purple), respectively. As described below, only the blue and purple sets

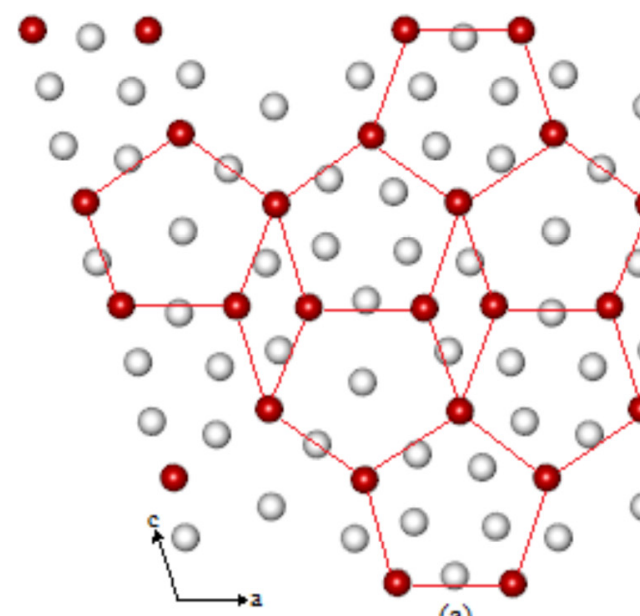

(a)

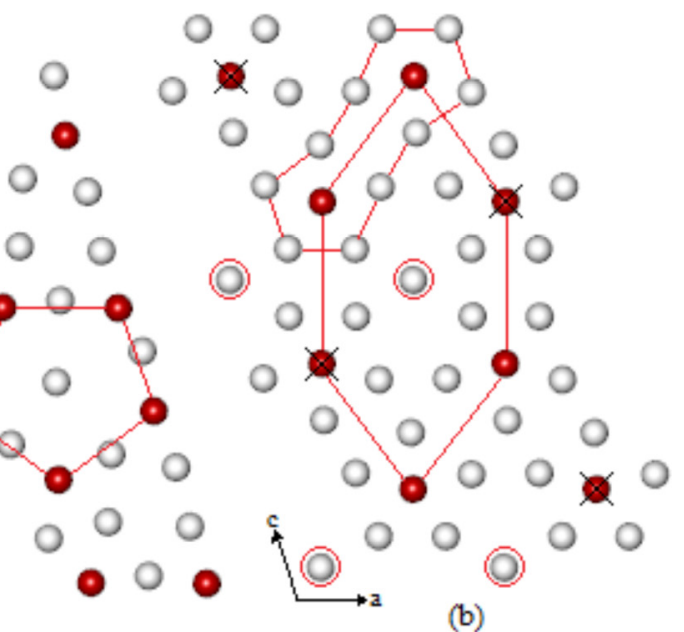

FIG. 2. (Color online) (a) The Fe atoms in the flat layers form rhombi and two types of pentagons. (b) The puckered layers consist of Fe atoms in the middle of bipentagonal Al motifs, connected by so-called glue atoms (circled). 


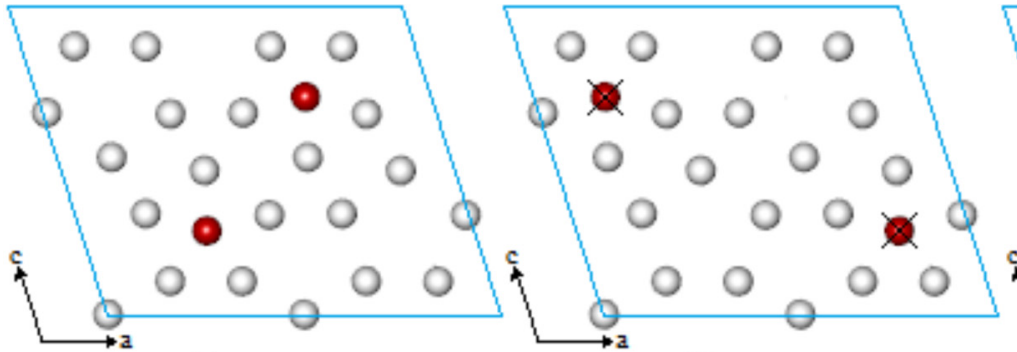

(a)

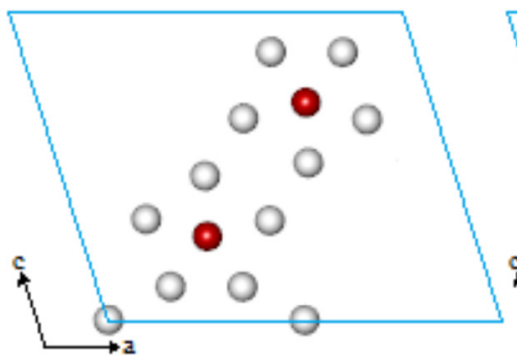

(d) (b)

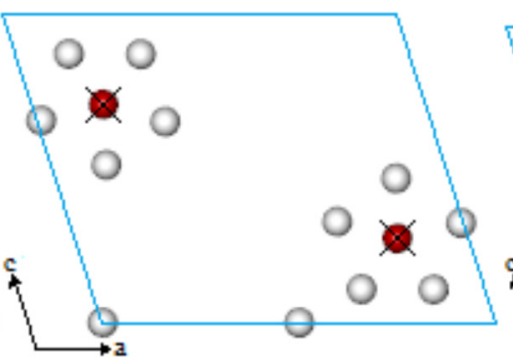

(e)

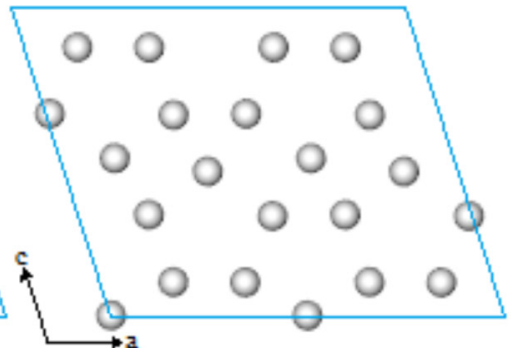

(c)

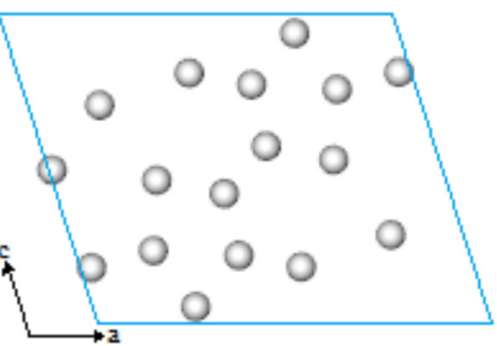

(f)

FIG. 3. (Color online) Incomplete variations in the bulk terminations used as trial structures in the calculations. (a) Puckered top, (b) puckered bottom, (c) puckered without Fe, (d) puckered incomplete $A$, (e) puckered incomplete $B$, (f) flat without Fe. The parallelogram in each case denotes the unit cell.

were used in the full structure analysis, giving a cumulative energy range of $2818 \mathrm{eV}$.

\section{B. Calculations}

The Barbieri-Van Hove SATLEED package was used for the dynamical LEED calculations, and the relativistic phase shifts were calculated with the phase shift package that comes bundled with it [14]. Four different sets of phase shifts were

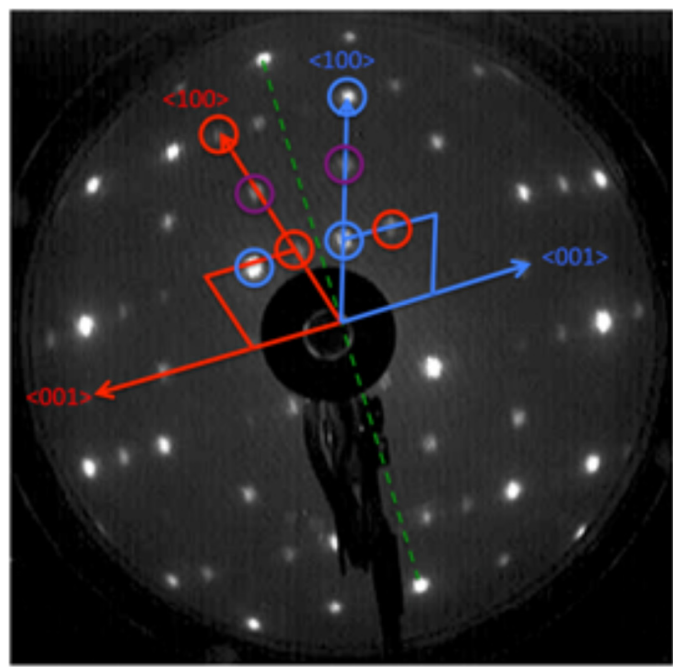

FIG. 4. (Color online) LEED pattern from $\mathrm{Al}_{13} \mathrm{Fe}_{4}(010)$ taken at $300 \mathrm{~K}$ with an electron-beam energy of $25 \mathrm{eV}$. The blue (pointing right) and red (pointing left) lines indicate reciprocal lattice vector directions for two twins, which are symmetrically equivalent to each other by the twinning mirror plane (green dashed line). The purple (darkest) circles represent LEED spots that are present for both twins. The reciprocal lattice unit cells in each case are denoted by parallelograms. used; one for $\mathrm{Al}$ atoms in the top layer, one for $\mathrm{Fe}$ atoms in the top layer, and the remaining two for the $\mathrm{Al}$ and $\mathrm{Fe}$ atoms in the deeper layers. The agreement between experimental and calculated data was quantified with Pendry's $R$ factor $R_{\mathrm{p}}$, and the error bars were calculated using the Pendry double- $R$ (RR) function [15]. At the beginning of the calculation, the Debye temperatures were set to the tabulated values of $470 \mathrm{~K}$ for $\mathrm{Fe}$ and $428 \mathrm{~K}$ for $\mathrm{Al}$. The potential was described by up to ten phase shifts, and the imaginary part of the inner potential set at $-5 \mathrm{eV}$. Optimizing these values and the real part of the inner potential was performed as the final stage of the analysis after the best-fit structure was determined.

The calculations were performed in Lappeenranta University of Technology using a 200-core calculation grid and software provided by Techila Technologies [16]. The calculation was split into 75 parts, each job running on a separate core. Depending on grid usage, this meant a completion time of approximately $50-100 \mathrm{~min}$ for the first part of the calculation during the final optimization of the structure.

We started with the blue and red datasets. The absolute intensities of beams related by symmetry were compared, which gave a relation of $4: 1$ for blue:red, indicating that the part of the surface surveyed in the LEED experiment had four times as much of the blue twin as the red twin. As shown later, this is consistent with the optimal mixing ratio obtained from the full LEED analysis.

To analyze the intensity data, a combination of the blue and purple datasets was used, and the red dataset was ignored due to its small size. Using this approximation meant not taking the effects of the red twin into account within the purple dataset intensities. Only the $z$ coordinates (perpendicular to the surface) were varied in the analysis. The analysis was also performed starting from the relaxed unit cell from the earlier density functional theory (DFT) analysis [8], but the difference between lattice parameters was only $0.43 \%$, 
TABLE I. Initial $R$ factors and the calculated Pendry variances for the trial structures. The purple and blue datasets were included in this analysis. The first, second, third, and fourth on the top row refer to the number of layers allowed to relax.

\begin{tabular}{lccccc}
\hline \hline Model & Unrelaxed & $\begin{array}{c}R \text {-factor } \\
\text { first }\end{array}$ & $\begin{array}{c}R \text {-factor } \\
\text { second }\end{array}$ & $\begin{array}{c}R \text {-factor } \\
\text { third }\end{array}$ & $\begin{array}{c}R \text {-factor } \\
\text { fourth }\end{array}$ \\
\hline$F$ & 0.84 & 0.71 & & & \\
$F$ without Fe & 0.88 & 0.77 & & & \\
$P$ & 0.88 & 0.76 & & & \\
$P$ no top Fe & 0.85 & 0.71 & & & \\
$P$ no bottom Fe & 0.89 & 0.67 & & & \\
$P$ without Fe & 0.85 & 0.73 & & & \\
$P$ incomplete $A$ & 0.87 & 0.55 & 0.37 & 0.30 & 0.29 \\
$P$ incomplete $B$ & 0.85 & 0.77 & & & \\
$P$ incomplete $A$ & 0.86 & 0.62 & & & \\
without Fe & & & & & \\
$P$ incomplete $B$ & 0.81 & 0.77 & & & \\
$\quad$ without Fe & & & & & \\
$P$ Pendry variance & & 0.06 & 0.04 & 0.03 & 0.03 \\
\hline \hline
\end{tabular}

so the results were essentially identical. In this case, the underlying $F$ and $P$ layers were no longer simply laterally shifted by $a / 2$. SATLEED does not support simultaneous calculation and optimization of more than one termination, so each of the $F$ and $P$ terminations was optimized separately and averaged as was performed by Shin et al. [17].

One concern for complex structures is to constrain the number of fitted parameters so that it does not exceed a certain number related to the size of the dataset used in the analysis. As a rule, this is taken to be about $50 \mathrm{eV}$ of data per parameter varied. The structure was allowed to relax down to the fourth layer. With a purple-blue combined energy range of $2818 \mathrm{eV}$ and 49 adjustable parameters, this gives approximately $57 \mathrm{eV}$ per parameter.

\section{Results}

The initial $R$ factors are listed in Table I for all the structural models considered in this analysis. Structures with a Pendry $R$ factor less than the sum of the lowest $R$ factor and the Pendry variance $(R R)$ were chosen for further analysis. After allowing the top layer to relax, the puckered incomplete $A$-structure model emerged as the best-fit structure with an $R$ factor of about 0.55 and a variance of 0.06 , ruling out all other models that were tested. This is the same model proposed by Ledieu

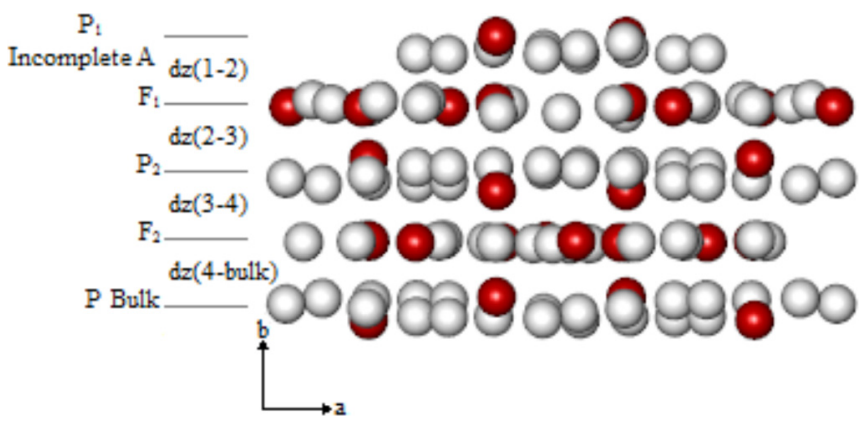

FIG. 5. (Color online) Side view of the relaxed best-fit structure. Average interlayer spacing is denoted by $d z$ (see Table II).
TABLE II. Average interlayer spacings $(d z)$ and rms puckering amplitudes $(\Delta)$ for the optimized best-fit termination. The bulk puckering is $0.30 \AA$ for the $P$ layers and $0.12 \AA$ for an incomplete $P$ layer.

\begin{tabular}{|c|c|c|c|}
\hline Parameter & $\begin{array}{c}\text { LEED } P \\
\text { incomplete } A(\AA)\end{array}$ & $\begin{array}{l}\text { DFT } P \text { incomplete } \\
A \text { (Ref. [8]) (̊) }\end{array}$ & Bulk $(\AA)$ \\
\hline$d z(1$ to 2$)$ & $1.68 \pm 0.02$ & 1.671 & 1.876 \\
\hline$d z(2$ to 3$)$ & $2.07 \pm 0.03$ & 2.032 & 2.007 \\
\hline$d z(3$ to 4$)$ & $2.01 \pm 0.04$ & 2.008 & 2.007 \\
\hline$d z(1$ bulk $)$ & $1.99 \pm 0.08$ & 2.010 & 2.007 \\
\hline$\Delta 1$ & 0.19 & 0.16 & 0.12 \\
\hline$\Delta 2$ & 0.12 & 0.08 & 0 \\
\hline$\Delta 3$ & 0.25 & 0.22 & 0.30 \\
\hline$\Delta 4$ & 0.02 & 0.01 & 0 \\
\hline
\end{tabular}

et al. [8]. Further optimization of this termination, relaxing up to four surface layers, produced an $R$ factor of 0.29 . After optimization of the nonstructural parameters, the $R$ factor was further reduced to 0.26 . The puckered incomplete $A$ model without the glue atoms was also tested, but this resulted in a negligibly small change in $R$ factor.

Figure 5 shows a side view of the best-fit puckered termination, and the relaxations are shown in Table II. The top layer resides slightly closer to the underlying flat layer, reducing the puckering amplitude, but causing puckering of the flat layer underneath. The puckered layer deeper in the structure exhibits a slightly smaller puckering amplitude compared to the $0.30-\AA$ pucker in the bulk layers. The structural parameters for the earlier DFT study (Ledieu et al., Ref. [8]) are also shown in Table II. It can be seen that the average layer relaxations from the LEED correspond closely to the DFT values. The average deviations between the individual atom positions are $0.04 \AA$ for the top (incomplete) $P_{1}$ layer, $0.11 \AA$ for the $F_{1}$ layer, and $0.07 \AA$ for the $P_{2}$ layer. The best-fit coordinates from both analyses are provided in the Supplemental Material [12].

After optimization, the intensities of those beams that belong to the purple dataset were used to find the optimal mixing ratio for the blue and red twins. This was performed by generating two calculated intensity sets; one was indexed based on the red twin, and the other was indexed based on the blue twin. These two intensity datasets were then mixed together in steps of $10 \%$. The final $R$ factors for the best-fit termination

TABLE III. $R$ factors for the optimized puckered incomplete $A$ termination with different mixing ratios for the blue and red twins.

\begin{tabular}{lc}
\hline \hline Mixing relation (blue:red) & $R_{\mathrm{p}}$ \\
\hline $100: 0$ & 0.263 \\
$90: 10$ & 0.242 \\
$\mathbf{8 0 : 2 0}$ & $\mathbf{0 . 2 3 4}$ \\
$70: 30$ & 0.236 \\
$60: 40$ & 0.243 \\
$50: 50$ & 0.255 \\
$40: 60$ & 0.271 \\
$30: 70$ & 0.292 \\
$20: 80$ & 0.319 \\
$10: 90$ & 0.351 \\
$0: 100$ & 0.396 \\
\hline
\end{tabular}



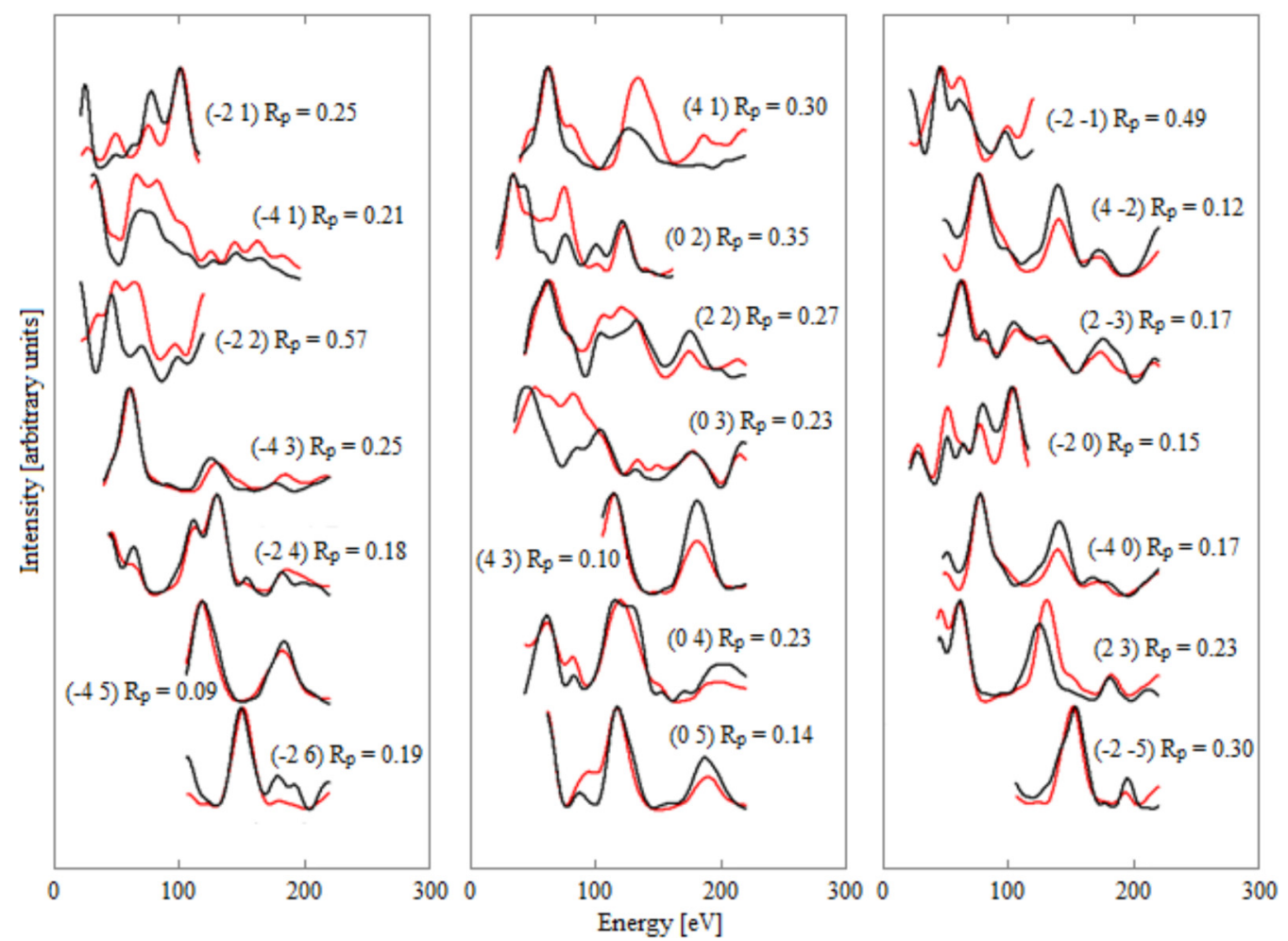

FIG. 6. (Color online) Experimental (black/bold) and calculated (red/light) LEED spectra for the optimized puckered incomplete $A$ termination.

after optimizations are given in Table III for different mixing ratios. The best fit was achieved with $80 \%$ of the blue twin and $20 \%$ of the red twin. The final $R$ factor after the mixing was 0.23 . The experimental and calculated spectra for the best-fit structure are shown in Fig. 6.

\section{DISCUSSION AND CONCLUSIONS}

The results of this study represent a step in elucidating the trends in the surface structures of these complex materials and how these materials become candidates for catalysts [18]. The results indicate that the top layer of the $\mathrm{Al}_{13} \mathrm{Fe}_{4}(010)$ surface is similar to a bulk puckered layer but lacks the Fe atoms below the mean plane position and the $\mathrm{Al}$ atoms that would surround them. This supports the previous STM and DFT analysis [8]. The incomplete top layer resides approximately $10 \%$ closer to the underlying flat layer, compared to a bulk structure, with an oscillating relaxation for the next few deeper layers. Based on the LEED analysis, the puckering amplitudes are slightly larger compared to the previous DFT results. There were not enough data to deduce lateral shifts of the atoms in this analysis. It was also not possible to verify the presence or absence of the connecting glue atoms, which may be indicative of large amplitudes of the motion of those atoms. In the DFT analysis, the model without the glue atoms was found to be energetically more favorable. However, some glue atoms were not fully desorbed during the annealing and were still present on the surface in the STM images [8].

The selection of the $P$ termination follows the trend established on other CMA surfaces to terminate in dense Al-rich layers, although the partial top layer is less common. For instance, this restructuring of the surface layer is quite different from the structure that was determined for the similar compound surface $\mathrm{Al}_{13} \mathrm{Co}_{4}$ (100) [17]. In that case, although the topmost surface layer was also found to be the Al-dense $P$ layer, it was essentially a simple truncation of the bulk structure with only the $\mathrm{Co}$ atoms missing. Conversely, the $\mathrm{Al}_{13} \mathrm{Fe}_{4}$ top layer is missing half of its bipentagons, which implies that only intact bipyramid clusters are stable at this surface. This difference was discussed in the recent STM/DFT study [8] and was attributed to the Al-Fe bonds being stronger than Al-Co bonds. The $\mathrm{Al}_{13} \mathrm{Fe}_{4}$ structure also does not exhibit inward and outward relaxations simultaneously, which was the case with $\mathrm{Al}_{13} \mathrm{Co}_{4}$.

The large top-layer relaxation found for the surface of $\mathrm{Al}_{13} \mathrm{Fe}_{4}$ is also unusual and is most likely a consequence of the absence of half of the top layer. Without the stabilizing influence of the full top layer, the remaining atoms in the top layer are pulled toward the underlying flat layer and as a consequence, produce a significant distortion in that layer. Although no contraction was found for the surface of $\mathrm{Al}_{13} \mathrm{Co}_{4}$ (100) [17], which terminates in a $P$ layer with the Co atoms removed, a recent study of a similar alloy surface with a partial top layer $\mathrm{Al}_{5} \mathrm{Co}_{2}(001)$ also found a large contraction of the top layer of about $10 \%$ [19].

\section{ACKNOWLEDGMENTS}

We thank N. A. Stanisha and J. S. Dulny for their assistance with extracting the LEED intensities. We thank the Academy of Finland Project No. 277829 and CNRS for the INCAS Project No. PICS05892 for financial support. This work was supported by the ANR-DFG CAPRICE 2011-INTB 1001-01 and granted access to the HPC resources of IDRIS under the allocation 2015096339. 
[1] M. Krajci and J. Hafner, Ab initio study of quasiperiodic monolayers on a fivefold i-AlPdMn surface, Phys. Rev. B 71, 184207 (2005).

[2] M. Krajci, J. Hafner, and M. Mihalkovic, Ab initio study of the surface of a decagonal Al-Co-Ni quasicrystal, Phys. Rev. B 73, 134203 (2006).

[3] T. Deniozou, R. Addou, A. K. Shukla, M. Heggen, M. Feuerbacher, M. Krajci, J. Hafner, R. Widmer, O. Gröning, V. Fournée, J. M. Dubois, and J. Ledieu, Structure of the (010) surface of the orthorhombic complex metallic alloy $\mathrm{T}_{-} \mathrm{Al}_{3}(\mathrm{Mn}, \mathrm{Pd})$, Phys. Rev. B 81, 125418 (2010).

[4] M. Armbrüster, K. Kovnir, Y. Grin, and R. Schlögl, in Complex Metallic Alloys: Fundamentals and Applications, edited by J.-M. Dubois and E. Belin-Ferré (Wiley-VCH, Weinheim, 2010), pp. 385-399.

[5] M. Armbrüster, K. Kovnir, M. Friedrich, D. Teschner, G. Wowsnick, M. Hahne, P. Gille, L. Szentmiklosi, M. Feuerbacher, M. Heggen, F. Girgsdies, D. Rosenthal, R. Schlögl, and Y. Grin, $\mathrm{Al}_{13} \mathrm{Fe}_{4}$ as a low-cost alternative for palladium in heterogeneous hydrogenation, Nature Mater. 11, 690 (2012).

[6] C. L. Henley, Current models of decagonal atomic structure, J. Non-Cryst. Solids 153-154, 172 (1993).

[7] P. Jeglič, S. Vrtnik, M. Bobnar, M. Klanjšek, B. Bauer, P. Gille, Y. Grin, F. Haarmann, and J. Dolinšek, M-Al-M groups trapped in cages of $\mathrm{Al}_{13} \mathrm{M}_{4}(\mathrm{M}=\mathrm{Co}, \mathrm{Fe}, \mathrm{Ni}, \mathrm{Ru})$ complex intermetallic phases as seen via NMR, Phys. Rev. B 82, 104201 (2010).

[8] J. Ledieu, É. Gaudry, L. N. Serkovic Loli, S. Alarcón Villaseca, M.-C. de Weerd, M. Hahne, P. Gille, Y. Grin, J.-M. Dubois, and V. Fournée, Structural Investigation of the (010) Surface of the $\mathrm{Al}_{13} \mathrm{Fe}_{4}$ Catalyst, Phys. Rev. Lett. 110, 076102 (2013).

[9] P. Gille and B. Bauer, Single crystal growth of $\mathrm{Al}_{13} \mathrm{Co}_{4}$ and $\mathrm{Al}_{13} \mathrm{Fe}_{4}$ from Al-rich solutions by the Czochralski method, Cryst. Res. Technol. 43, 1161 (2008).
[10] A. Mayer, H. Salopaasi, K. Pussi, and R. D. Diehl, A novel method for the extraction of intensity - energy spectra from lowenergy electron diffraction patterns, Comput. Phys. Commun. 183, 1443 (2012).

[11] A. Mayer, H. Salopaasi, N. Ferralis, K. Pussi, and R. D. Diehl, http://andim.github.io/easyleed/ (2015).

[12] See Supplemental Material at http://link.aps.org/supplemental/ 10.1103/PhysRevB.92.014109 for experimental data and best-fit coordinates.

[13] K. Saito, K. Sugiyama, and K. Hiraga, $\mathrm{Al}_{13} \mathrm{M}_{4}$-type structures and atomic models of their twins, Mater. Sci. Eng., A 294, 279 (2000).

[14] A. Barbieri and M. A. Van Hove, http://www.icts.hkbu. edu.hk/surfstructinfo/SurfStrucInfo_files/leed/leedpack.html (2015).

[15] J. B. Pendry, Reliability factors for LEED calculations, J. Phys. C: Solid State Phys. 13, 937 (1980).

[16] Techila Technologies Ltd., http://www.techilatechnologies.com (2014).

[17] H. Shin, K. Pussi, É. Gaudry, J. Ledieu, V. Fournée, S. Alarcón Villaseca, J.-M. Dubois, Y. Grin, P. Gille, W. Moritz, and R. D. Diehl, Structure of the orthorhombic $\mathrm{Al}_{13} \mathrm{Co}_{4}(100)$ surface using LEED, STM, and $a b$ initio studies, Phys. Rev. B 84, 085411 (2011).

[18] L. Piccolo, $\mathrm{Al}_{13} \mathrm{Fe}_{4}$ selectively catalyzes the hydrogenation of butadiene at room temperature, Chem. Commun. 49, 9149 (2013).

[19] M. Meier, J. Ledieu, M.-C. De Weerd, Y.-T. Huang, G. J. P. Abreu, K. Pussi, R. D. Diehl, T. Mazet, V. Fournée and É. Gaudry, Interplay between bulk atomic clusters and surface structure in complex intermetallic compounds: The case study of the $\mathrm{Al}_{5} \mathrm{Co}_{2}(001)$ surface, Phys. Rev. B 91, 085414 (2015). 